\title{
Physical properties of the WASP-67 planetary system from multi-colour photometry $\star, \star \star$
}

L. Mancini ${ }^{1}$, J. Southworth ${ }^{2}$, S. Ciceri ${ }^{1}$, S. Calchi Novati ${ }^{3,4}$, M. Dominik ${ }^{5}$, Th. Henning ${ }^{1}$, U. G. Jørgensen ${ }^{6,7}$, H. Korhonen ${ }^{8,6,7}$, N. Nikolov ${ }^{9}$, K. A. Alsubai ${ }^{10}$, V. Bozza ${ }^{4,11}$, D. M. Bramich ${ }^{12}$, G. D’Ago ${ }^{4,11}$, R. Figuera Jaimes ${ }^{5,13}$, P. Galianni ${ }^{5}$, S.-H. Gu ${ }^{14,15}$, K. Harpsøe $\mathrm{e}^{6,7}$, T. C. Hinse ${ }^{16}$, M. Hundertmark ${ }^{5}$, D. Juncher ${ }^{6,7}$, N. Kains ${ }^{17}$, A. Popovas ${ }^{6,7}$, M. Rabus ${ }^{18,1}$, S. Rahvar ${ }^{19}$, J. Skottfelt ${ }^{6,7}$, C. Snodgrass ${ }^{20}$, R. Street ${ }^{21}$, J. Surdej ${ }^{22}$, Y. Tsapras ${ }^{21,23}$, C. Vilela ${ }^{2}$, X.-B. Wang ${ }^{14,15}$, and O. Wertz ${ }^{22}$

${ }^{1}$ Max-Planck Institute for Astronomy, Königstuhl 17, 69117 Heidelberg, Germany e-mail: mancini@mpia.de

2 Astrophysics Group, Keele University, Staffordshire, ST5 5BG, UK

3 International Institute for Advanced Scientific Studies (IIASS), 84019 Vietri Sul Mare (SA), Italy

${ }^{4}$ Department of Physics, University of Salerno, Via Giovanni Paolo II, 84084 Fisciano, Italy

5 SUPA, University of St Andrews, School of Physics \& Astronomy, North Haugh, St Andrews, KY16 9SS, UK

${ }^{6}$ Niels Bohr Institute, University of Copenhagen, Juliane Maries vej 30, 2100 Copenhagen Ø, Denmark

7 Centre for Star and Planet Formation, Geological Museum, Øster Voldgade 5-7, 1350 Copenhagen, Denmark

${ }^{8}$ Finnish Centre for Astronomy with ESO (FINCA), University of Turku, Väisäläntie 20, 21500 Piikkiö, Finland

9 Astrophysics Group, University of Exeter, Stocker Road, EX4 4QL, Exeter, UK

10 Qatar Foundation, PO Box 5825, Doha, Qatar

11 Istituto Nazionale di Fisica Nucleare (INFN), Sezione di Napoli, 80126 Napoli, Italy

12 Qatar Environment and Energy Research Institute, Qatar Foundation, Tornado Tower, Floor 19, PO Box 5825, Doha, Qatar

13 European Southern Observatory, Karl-Schwarzschild-Straße 2, 85748 Garching bei München, Germany

14 Yunnan Observatories, Chinese Academy of Sciences, 650011 Kunming, PR China

15 Key Laboratory for the Structure and Evolution of Celestial Objects, Chinese Academy of Sciences, 650011 Kunming, PR China

16 Korea Astronomy and Space Science Institute, Daejeon 305-348, Republic of Korea

17 Space Telescope Science Institute, 3700 San Martin Drive, Baltimore, MD 21218, USA

18 Instituto de Astrofísica, Facultad de Física, Pontificia Universidad Católica de Chile, Av. Vicuña Mackenna 4860, 7820436 Macul, Santiago, Chile

19 Department of Physics, Sharif University of Technology, PO Box 11155-9161 Tehran, Iran

${ }_{20}$ Max Planck Institute for Solar System Research, Justus-von-Liebig-Weg 3, 37077 Göttingen, Germany

21 Las Cumbres Observatory Global Telescope Network, 6740B Cortona Drive, Goleta, CA 93117, USA

22 Institut d'Astrophysique et de Géophysique, Université de Liège, 4000 Liège, Belgium

23 School of Physics and Astronomy, Queen Mary University of London, Mile End Road, London, E1 4NS, UK

Received 30 April 2014 / Accepted 28 June 2014

\section{ABSTRACT}

Context. The extrasolar planet WASP-67 b is the first hot Jupiter definitively known to undergo only partial eclipses. The lack of the second and third contact points in this planetary system makes it difficult to obtain accurate measurements of its physical parameters. Aims. By using new high-precision photometric data, we confirm that WASP-67 b shows grazing eclipses and compute accurate estimates of the physical properties of the planet and its parent star.

Methods. We present high-quality, multi-colour, broad-band photometric observations comprising five light curves covering two transit events, obtained using two medium-class telescopes and the telescope-defocusing technique. One transit was observed through a Bessel- $R$ filter and the other simultaneously through filters similar to Sloan $g^{\prime} r^{\prime} i^{\prime} z^{\prime}$. We modelled these data using JKTEBOP. The physical parameters of the system were obtained from the analysis of these light curves and from published spectroscopic measurements. Results. All five of our light curves satisfy the criterion for being grazing eclipses. We revise the physical parameters of the whole WASP-67 system and, in particular, significantly improve the measurements of the planet's radius $\left(R_{\mathrm{b}}=1.091 \pm 0.046 R_{\mathrm{Jup}}\right)$ and density $\left(\rho_{\mathrm{b}}=0.292 \pm 0.036 \rho_{\text {Jup }}\right)$, as compared to the values in the discovery paper $\left(R_{\mathrm{b}}=1.4_{-0.2}^{+0.3} R_{\text {Jup }}\right.$ and $\left.\rho_{\mathrm{b}}=0.16 \pm 0.08 \rho_{\text {Jup }}\right)$. The transit ephemeris was also substantially refined. We investigated the variation of the planet's radius as a function of the wavelength, using the simultaneous multi-band data, finding that our measurements are consistent with a flat spectrum to within the experimental uncertainties.

Key words. planetary systems - stars: fundamental parameters - techniques: photometric

\footnotetext{
* Based on data collected with GROND at the MPG $2.2 \mathrm{~m}$ telescope and DFOSC at the Danish $1.54 \mathrm{~m}$ telescope.

$\star \star$ Full Table 2 is only available at the CDS via anonymous ftp to cdsarc.u-strasbg. fr (130.79.128.5) or via http://cdsarc.u-strasbg.fr/viz-bin/qcat?]/A+A/568/A127
}

\section{Introduction}

WASP-67 b (Hellier et al. 2012) is a transiting extrasolar planet (TEP), discovered by the SuperWASP group (Pollacco et al. 2006), orbiting a $\mathrm{K} 0 \mathrm{~V}$ star $(V=12.5 \mathrm{mag})$ every $4.61 \mathrm{~d}$. It is 
Table 1. Details of the transit observations presented in this work.

\begin{tabular}{lccccccccccc}
\hline \hline Instrument & $\begin{array}{c}\text { Date of } \\
\text { first obs. }\end{array}$ & $\begin{array}{c}\text { Start time } \\
(\mathrm{UT})\end{array}$ & $\begin{array}{c}\text { End time } \\
(\mathrm{UT})\end{array}$ & $N_{\mathrm{obs}}$ & $\begin{array}{c}T_{\exp } \\
(\mathrm{s})\end{array}$ & $\begin{array}{c}T_{\mathrm{obs}} \\
(\mathrm{s})\end{array}$ & Filter & Airmass & $\begin{array}{c}\text { Moon } \\
\text { illum. }\end{array}$ & $\begin{array}{c}\text { Aperture } \\
\text { radii }(\mathrm{px})\end{array}$ & $\begin{array}{c}\text { Scatter } \\
(\mathrm{mmag})\end{array}$ \\
\hline GROND & 20120604 & $03: 00$ & $10: 50$ & 162 & $70 / 90$ & $110 / 120$ & Sloan $g^{\prime}$ & $2.14 \rightarrow 1.01 \rightarrow 1.22$ & $98 \%$ & $34,50,80$ & 1.08 \\
GROND & 20120604 & $03: 00$ & $10: 50$ & 162 & $70 / 90$ & $110 / 120$ & Sloan $r^{\prime}$ & $2.14 \rightarrow 1.01 \rightarrow 1.22$ & $98 \%$ & $38,60,85$ & 0.56 \\
GROND & 20120604 & $03: 00$ & $10: 50$ & 162 & $70 / 90$ & $110 / 120$ & Sloan $i^{\prime}$ & $2.14 \rightarrow 1.01 \rightarrow 1.22$ & $98 \%$ & $40,60,85$ & 0.72 \\
GROND & 20120604 & $03: 00$ & $10: 50$ & 162 & $70 / 90$ & $110 / 120$ & Sloan $z^{\prime}$ & $2.14 \rightarrow 1.01 \rightarrow 1.22$ & $98 \%$ & $40,60,85$ & 0.64 \\
DFOSC & 20130622 & $04: 30$ & $08: 33$ & 136 & 100 & 110 & Bessel $R$ & $1.12 \rightarrow 1.01 \rightarrow 1.17$ & $97 \%$ & $20,35,55$ & 0.48 \\
\hline
\end{tabular}

Notes. $N_{\mathrm{obs}}$ is the number of observations, $T_{\text {exp }}$ is the exposure time, $T_{\mathrm{obs}}$ is the observational cadence, and "Moon illum." is the fractional illumination of the Moon at the midpoint of the transit. The aperture sizes are the radii of the software apertures for the star, inner sky and outer sky, respectively. Scatter is the rms scatter of the data versus a fitted model.

an inflated $\left(\rho_{\mathrm{b}} \ll \rho_{\text {Jup }}\right)$ hot Jupiter $(a \sim 0.05 \mathrm{AU})$ on a grazing orbit (impact parameter $b>0.9$ ), causing the transit light curve to have an atypical V shape. Hellier et al. (2012) found that WASP-67 b satisfies the grazing criterion $\left(X=b+R_{\mathrm{b}} / R_{\star}>1\right)$ by $3 \sigma$, which makes it the first TEP definitively known to have a grazing eclipse ${ }^{1}$. In this particular configuration, the second and third contact points (e.g. Winn 2010) are missing and the light curve solution becomes degenerate. This hampers accurate measurements of the photometric parameters of the system. Consequently, Hellier et al. (2012) measured the radius of the planet with a large uncertainty of $\sim 20 \%$. In such cases, highquality light curves are mandatory to reduce the error bars to levels similar to those of other known TEPs.

Here, we present the first photometric follow-up study of WASP-67 since its discovery paper. The main aim of this study is to refine the physical parameters of the system and ephemeris, setting the stage for a more detailed study in the near future. WASP-67 is located in field \#7 of the K2 phase of the NASA's Kepler mission ${ }^{2}$ and will be observed continuously for approximately $80 \mathrm{~d}$ in late 2015 .

\section{Observations and data reduction}

A complete transit of WASP-67 b was observed on 2012 June 4 (Table 1) using the Gamma Ray burst Optical and Near-infrared Detector (GROND) instrument mounted on the $\mathrm{MPG}^{3} 2.2 \mathrm{~m}$ telescope, which is located at the ESO observatory in La Silla (Chile). GROND is an imaging system capable of simultaneous photometric observations in four optical (similar to Sloan $\left.g^{\prime}, r^{\prime}, i^{\prime}, z^{\prime}\right)$ and three $\operatorname{NIR}(J, H, K)$ passbands (Greiner et al. 2008). Each of the four optical channels is equipped with a back-illuminated $2048 \times 2048$ E2V CCD with a field of view of $5.4^{\prime} \times 5.4^{\prime}$ at $0.158^{\prime \prime}$ pixel $^{-1}$. The three NIR channels use $1024 \times 1024$ Rockwell HAWAII-1 arrays with a field of view of $10^{\prime} \times 10^{\prime}$ at $0.6^{\prime \prime}$ pixel $^{-1}$. The telescope was autoguided during the observations, which were performed with the defocusing technique (Southworth et al. 2009).

Another complete transit of WASP-67 b was observed on 2013 June 22 by using the DFOSC imager mounted on the $1.54 \mathrm{~m}$ Danish Telescope, which is also at the ESO observatory in La Silla, during the 2013 observing campaign by the MiNDSTEp consortium (Dominik et al. 2010). The instrument has a E2V44-82 CCD camera with a field of view of $13.7^{\prime} \times 13.7^{\prime}$ and a plate scale of $0.39^{\prime \prime} \mathrm{pixel}^{-1}$. The observations

1 Other TEPs which might undergo grazing eclipses are WASP-34 (Smalley et al. 2011) and HAT-P-27/WASP-40 (Béky et al. 2011; Anderson et al. 2011).

2 http://keplerscience.arc.nasa.gov/K2/

3 Max Planck Gesellschaft.
Table 2. Excerpts of the light curves of WASP-67.

\begin{tabular}{lccrc}
\hline \hline Telescope & Filter & BJD (TDB) & Diff. mag. & Uncertainty \\
\hline ESO $2.2 \mathrm{~m}$ & $g^{\prime}$ & 2456082.655745 & 0.00061 & 0.00043 \\
ESO $2.2 \mathrm{~m}$ & $g^{\prime}$ & 2456082.657102 & 0.00142 & 0.00043 \\
ESO $2.2 \mathrm{~m}$ & $r^{\prime}$ & 2456082.655745 & 0.00083 & 0.00038 \\
ESO $2.2 \mathrm{~m}$ & $r^{\prime}$ & 2456082.657102 & 0.00101 & 0.00033 \\
ESO $2.2 \mathrm{~m}$ & $i^{\prime}$ & 2456082.655745 & 0.00069 & 0.00041 \\
ESO $2.2 \mathrm{~m}$ & $i^{\prime}$ & 2456082.657102 & 0.00117 & 0.00043 \\
ESO $2.2 \mathrm{~m}$ & $z^{\prime}$ & 2456082.653032 & -0.00041 & 0.00048 \\
ESO $2.2 \mathrm{~m}$ & $z^{\prime}$ & 2456082.654390 & -0.00117 & 0.00048 \\
DK $1.54 \mathrm{~m}$ & $R$ & 2456465.694278 & 0.00066 & 0.00141 \\
DK $1.54 \mathrm{~m}$ & $R$ & 2456465.695528 & 0.00033 & 0.00141 \\
\hline
\end{tabular}

Notes. This table is available at the CDS. A portion is shown here for guidance regarding its form and content.

were performed through a Bessel $R$ filter; the telescope was defocused and autoguided, and the CCD was windowed to reduce the readout time. With the applied defocus, the diameter of the PSF of the target and reference stars was $\sim 12^{\prime \prime}$, which is similar to that for the GROND images.

The optical data collected from both telescopes were reduced using DEFOT, an IDL ${ }^{4}$ pipeline for time-series photometry (Southworth et al. 2009). The images were debiased and flatfielded using standard methods and then subjected to aperture photometry using the APER ${ }^{5}$ task and an optimal ensemble of comparison stars. Pointing variations were followed by crosscorrelating each image against a reference image. The shape of the light curve is very insensitive to the aperture sizes, so we chose those that yielded the lowest scatter. The relative weights of the comparison stars were optimised simultaneously with a de-trending of the light curve to remove slow instrumental and astrophysical trends. This was achieved by fitting a straight line to the out-of-transit data for the DFOSC data and with a fourthorder polynomial for the GROND data (to compensate for the lack of reference stars caused by the smaller field of view).

The final differential-flux light curves are plotted in Fig. 1 and tabulated in Table 2. In particular, the GROND light curves in the top panel of Fig. 1 are reported superimposed to highlight the differences of the light-curve shape and the transit depth along the four passbands. Contrary to what is expected for higher-inclination systems (e.g. Knutson et al. 2007), the transit depth gradually increases moving from blue to red bands. This phenomenon happens because the planet only covers the limb of

\footnotetext{
4 IDL is a trademark of the ITT Visual Information Solutions: http://www . ittvis . com/ProductServices/IDL . aspx

5 APER is part of the ASTROLIB subroutine library distributed by NASA on http: //idlastro.gsfc.nasa.gov
} 

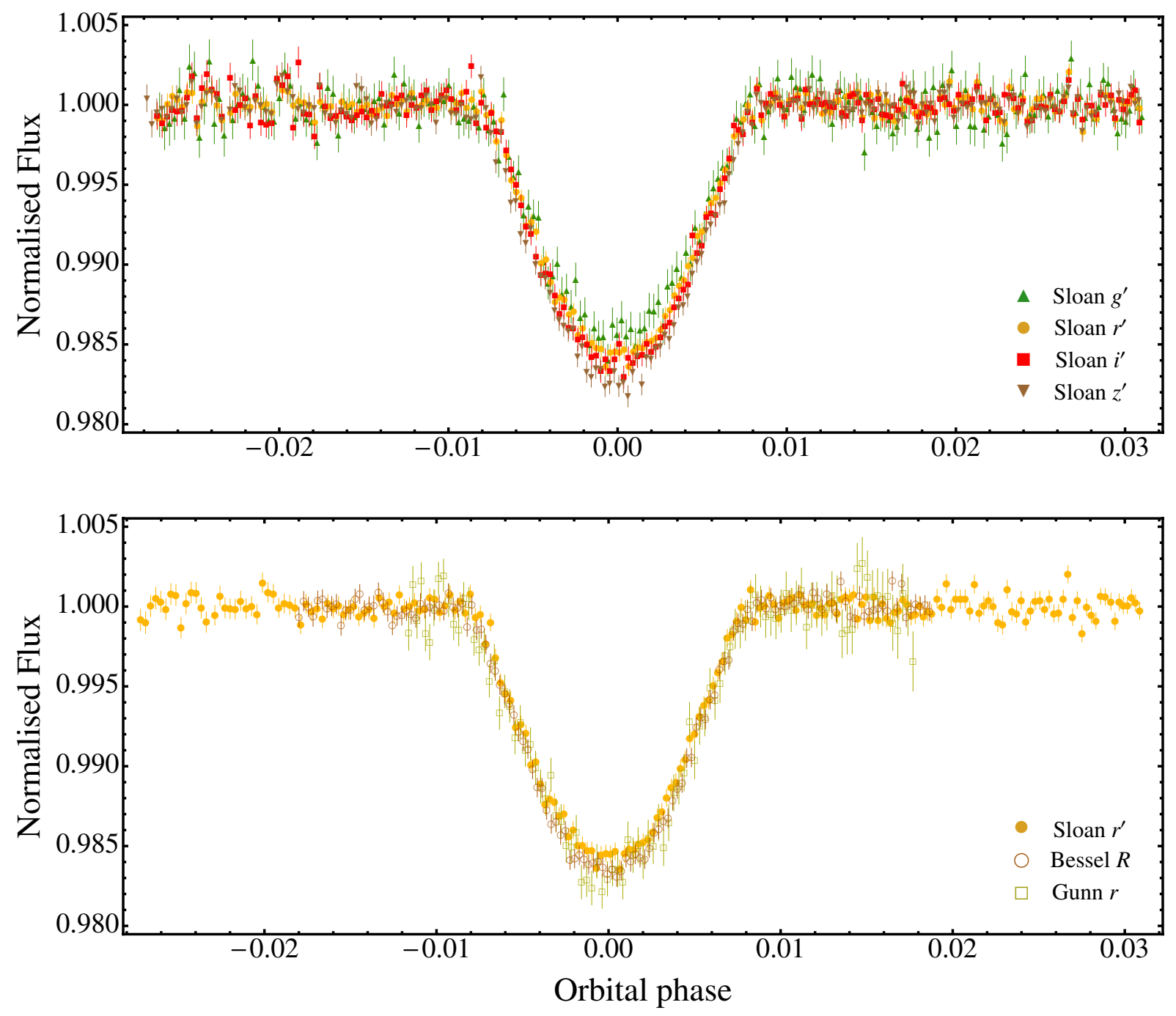

Fig. 1. Light curves of WASP-67b eclipses. Top panel: light curves obtained with GROND in $g^{\prime} r^{\prime} i^{\prime} z^{\prime}$, showing how the transit light curve shape changes with wavelength. The transit in the $g^{\prime}$ band is shallower than the other bands, as expected for a grazing eclipse, as limb darkening is stronger at bluer wavelengths. Bottom panel: light curves obtained with DFOSC in the $R$-band (June 2013, brown open circles), with GROND in the $r^{\prime}$-band (June 2012, yellow points) and with the Euler $1.2 \mathrm{~m}$ telescope in the $r$-band (July 2011, green open squares, Hellier et al. 2012). The light curves are superimposed to highlight variations in transit shape between the three measurements.

the star (as this is a grazing eclipse), which is fainter in the blue part of the optical spectrum than the red one due to the stronger limb darkening. Thus, we expect to see shallower eclipses in the bluest bands for this system.

The DFOSC Bessel- $R$ light curve is shown in the bottom panel of Fig. 1 and is superimposed with the GROND Sloan- $r^{\prime}$ light curve and that from Hellier et al. (2012), which was obtained with the Euler $1.2 \mathrm{~m}$ telescope through a Gunn- $r$ filter. This panel highlights the slight variation of the transit depth between the DFOSC and GROND light curves; the Euler data are more scattered and agree with both. Slight differences can be caused by the different filters used or by unocculted starspots. The latter hypothesis suggests a variation of the starspot activity of the WASP-67 A during a period of two years, which is reasonable for a $5200 \mathrm{~K}$ star.

Similar to some previous cases (Nikolov et al. 2012; Mancini et al. 2013b, 2014), the quality of the GROND NIR data were not good enough to extract usable photometry. We were only able to obtain a noisy light curve in the $J$ band that, which if we consider the particular transit geometry of the WASP-67 system, returned very inaccurate estimates of the photometric parameters in the light-curve fitting process (see next section) in comparison with the optical ones.

\section{Light-curve analysis}

Our light curves were modelled using the JKTEBOP ${ }^{6}$ code (see Southworth 2012, and references therein), which represents the star and planet as biaxial spheroids for the calculation of the reflection and ellipsoidal effects and as spheres for calculation of the eclipse shapes. The main parameters fitted by JKTEBOP are the orbital inclination, $i$, the transit midpoint, $T_{0}$, and the sum and ratio of the fractional radii of the star and planet, $r_{\mathrm{A}}+r_{\mathrm{b}}$ and $k=r_{\mathrm{b}} / r_{\mathrm{A}}$. The fractional radii are defined as $r_{\mathrm{A}}=R_{\mathrm{A}} / a$ and $r_{\mathrm{b}}=R_{\mathrm{b}} / a$, where $a$ is the orbital semimajor axis, and $R_{\mathrm{A}}$ and $R_{\mathrm{b}}$ are the absolute radii of the star and the planet, respectively.

Each light curve was analysed separately, using a quadratic law to model the limb darkening (LD) effect. Due to the difficulty of measuring accurate LD coefficients in TEP systems with impact parameters $b \geq 0.8$ (Müller et al. 2013), the WASP-67 A LD

6 The source code of JKTEBOP is available at http://www. astro. keele.ac.uk/jkt/codes/jktebop.html 


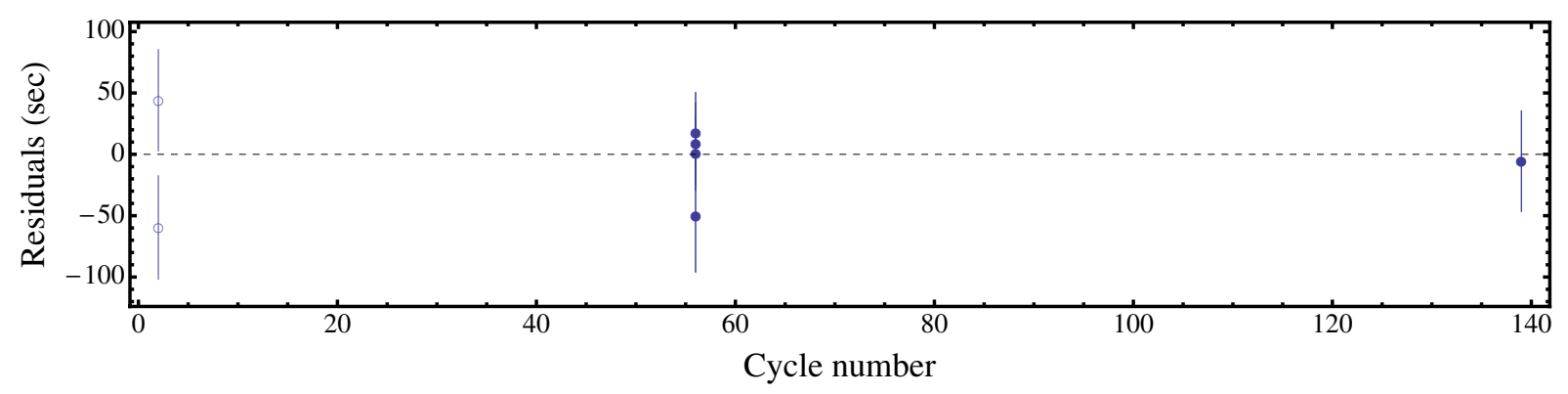

Fig. 2. Residuals for the timings of WASP-67 b at mid-transit versus a linear ephemeris. The two timings, based on the observations reported by Hellier et al. (2012), are plotted using open circles, while the other timings (this work) are plotted with filled circles.

Table 3. Times of WASP-67 $\mathrm{b}$ at mid-transit and their residuals versus a linear orbital ephemeris.

\begin{tabular}{lrcc}
\hline \hline $\begin{array}{l}\text { Time of minimum } \\
\text { BJD(TDB) }-2400000\end{array}$ & $\begin{array}{r}\text { Cycle } \\
\text { No. }\end{array}$ & $\begin{array}{c}\text { Residual } \\
\text { (d) }\end{array}$ & Reference \\
\hline $55833.60357 \pm 0.00032$ & 2 & 0.000510 & 1 \\
$55833.60237 \pm 0.00033$ & 2 & -0.000690 & 2 \\
$56082.78067 \pm 0.00034$ & 56 & -0.000578 & 3 \\
$56082.78126 \pm 0.00016$ & 56 & 0.000012 & 4 \\
$56082.78135 \pm 0.00019$ & 56 & 0.000102 & 5 \\
$56082.78145 \pm 0.00019$ & 56 & 0.000202 & 6 \\
$56465.77729 \pm 0.00016$ & 139 & -0.000064 & 7 \\
\hline
\end{tabular}

Notes. References: (1) Euler $1.2 \mathrm{~m}$ telescope (Hellier et al. 2012); (2) Trappist 0.6-m telescope (Hellier et al. 2012); (3) GROND $g^{\prime}$-band (this work); (4) GROND $r^{\prime}$-band (this work); (5) GROND $i^{\prime}$-band (this work); (6) GROND $z^{\prime}$-band (this work); (7) Danish 1.52-m telescope (this work)

coefficients were fixed to their theoretical values (Claret 2004b). We also assumed that the planetary orbit is circular (Hellier et al. 2012). We included the coefficients of a linear (DFOSC) or fourth (GROND) polynomial versus time in the fits to fully account for the uncertainty in the de-trending of the light curves.

We also considered the two light curves obtained with the Euler $1.2 \mathrm{~m}$ and Trappist $0.6 \mathrm{~m}$ telescopes, which were reported in Hellier et al. (2012). To present a homogeneous analysis, we re-fitted these two light curves using JKTEBOP in the same manner as for our own data.

As in previous works (Mancini et al. 2013a,b,c, 2014), we enlarged the error bars of the light curve points generated by our reduction pipeline. Such a process is necessary because the APER algorithm, which is used to perform aperture photometry, tends to underestimate the true uncertainties in the relative magnitude measurements. This is a typical situation in time-series photometry, where additional noise sources, such as red noise, are not accounted for by standard error-estimation algorithms (e.g. Carter \& Winn 2009). We, therefore, rescaled the error bars for each eclipse to give a reduced $\chi^{2}$ of $\chi_{v}^{2}=1$ and then again by using the $\beta$ approach (e.g. Gillon et al. 2006; Winn et al. 2008; Gibson et al. 2008).

\subsection{Orbital period determination}

We used our photometric data and those coming from the discovery paper (Hellier et al. 2012) to refine the orbital period of WASP-67 b. The transit time for each of the datasets was obtained by fitting with JKTEBOP, and uncertainties were estimated using Monte Carlo simulations. All timings were placed on the BJD(TDB) time system and are summarised in Table 3. The plot of the residuals is shown in Fig. 2. The resulting measurements of transit midpoints were fitted with a straight line to obtain a final orbital ephemeris:

$$
T_{0}=\mathrm{BJD}(\mathrm{TDB}) 2455824.37424(22)+4.6144109(27) E,
$$

where $E$ is the number of orbital cycles after the reference epoch, which we take to be that estimated by Hellier et al. (2012), and quantities in brackets denote the uncertainty in the final digit of the preceding number. The quality of fit, $\chi_{v}^{2}=1.90$, indicates that a linear ephemeris is not a perfect match to the observations. However, considering that our timings cover only three epochs, it is difficult to claim systematic deviations from the predicted transit times. Future Kepler data will enlarge the number of observed transit events of WASP-67 b and may rule in or out possible transit timing variations.

\subsection{Photometric parameters}

The GROND light curves and the JKTEBOP best-fitting models are shown in Fig. 3. A similar plot is reported in Fig. 4 for the light curves from the Danish Telescope and Hellier et al. (2012). The parameters of the fits are given in Table 4. Uncertainties in the fitted parameters from each solution were calculated from 5500 Monte Carlo simulations and by a residual-permutation algorithm (Southworth 2008). The larger of the two possible error bars was adopted for each case. The error bars for the fits to individual light curves are often strongly asymmetric due to the morphology of the light curve. The final photometric parameters were therefore calculated by multiplying the probability density functions of the different values. This procedure yielded error bars, which are close to symmetric for all photometric parameters, and are given in Table 4. The values obtained by Hellier et al. (2012) are also reported for comparison. Due to their lower quality, we did not use any of the GROND-NIR light curves to estimate the final photometric parameters of WASP-67.

\section{Physical properties}

Similarly to the Homogeneous Studies approach (Southworth 2012, and references therein), we used the photometric parameters estimated in the previous section and the spectroscopic properties of the parent star (velocity amplitude $K_{\mathrm{A}}=0.056 \pm$ $0.004 \mathrm{~km} \mathrm{~s}^{-1}$, effective temperature $T_{\text {eff }}=5200 \pm 100 \mathrm{~K}$, and metallicity $\left[\frac{\mathrm{Fe}}{\mathrm{H}}\right]=-0.07 \pm 0.09$; Hellier et al. 2012), to revise the physical properties of the WASP-67 system using the ABSDIM code.

We iteratively determined the velocity amplitude of the planet $\left(K_{\mathrm{b}}\right)$, which yielded the best agreement between the measured $r_{\mathrm{A}}$ and $T_{\text {eff }}$, and the values of $R_{\mathrm{A}} / a$ and $T_{\text {eff }}$ predicted by a 
L. Mancini et al.: Physical properties of WASP-67 b
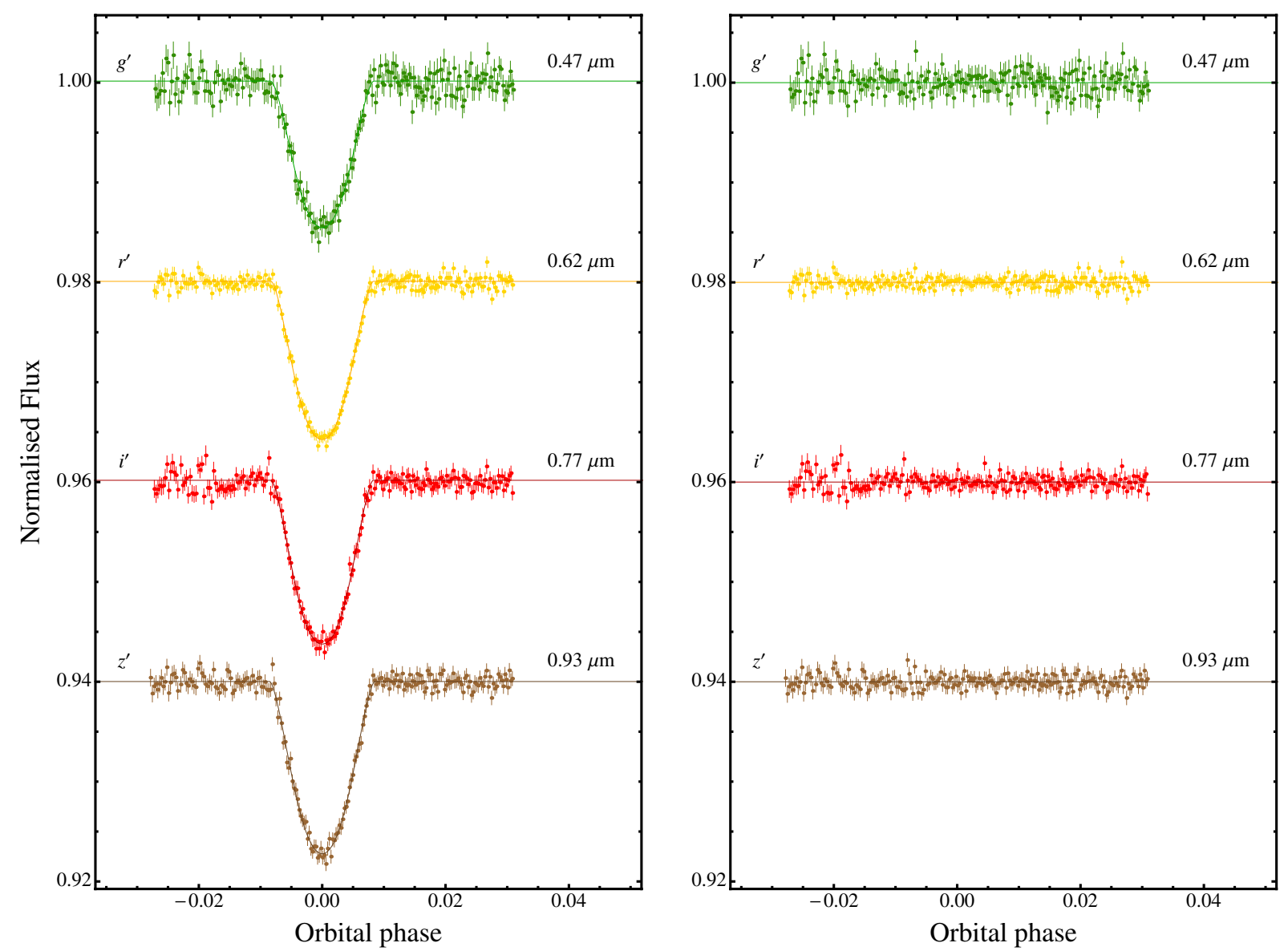

Fig. 3. Left-hand panel: simultaneous optical light curves of the WASP-67 eclipse observed with GROND. The JKTEBOP best fits are shown as solid lines for each optical data set. The passbands are labelled on the left of the figure, and their central wavelengths are given on the right. Right-hand panel: residuals of each fit.
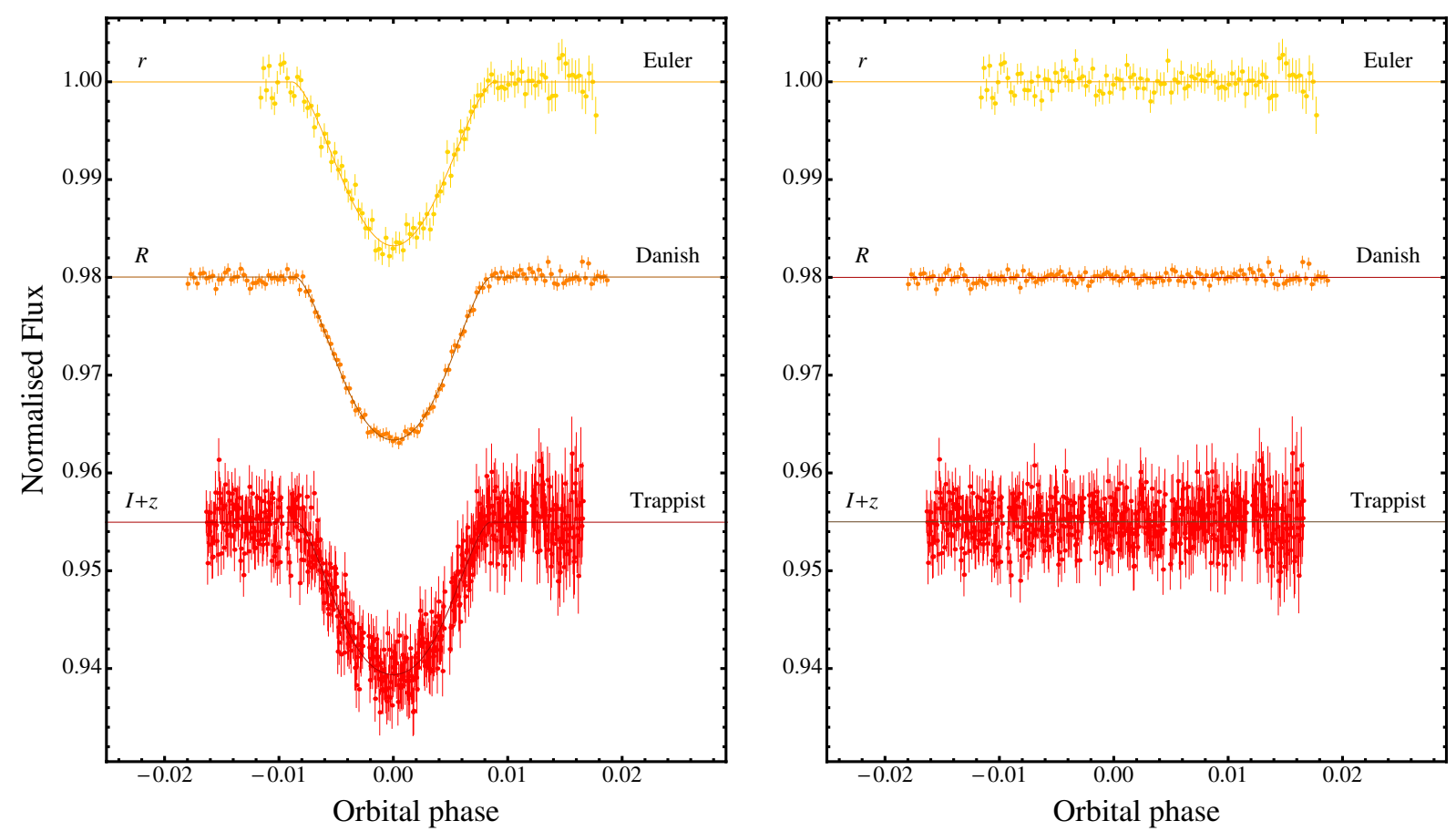

Fig. 4. Left-hand panel: light curves of the WASP-67 eclipses observed in Gunn- $r$ with the Euler telescope (Hellier et al. 2012), in Bessell- $R$ with the Danish telescope (this work) and with an $I+z$ filter with the TRAPPIST telescope (Hellier et al. 2012). The filters and the name of each telescope are labelled on the figure. The JKTEBOP best fits are shown as solid lines for each optical dataset. Right-hand panel: residuals of each fit. 
Table 4. Parameters of the JKTEBOP fits to the light curves of WASP-67.

\begin{tabular}{|c|c|c|c|c|c|c|}
\hline Telescope & Filter & $r_{\mathrm{A}}+r_{\mathrm{b}}$ & $k$ & $i^{\circ}$ & $r_{\mathrm{A}}$ & $r_{\mathrm{b}}$ \\
\hline MPG $2.2 \mathrm{~m}$ & Sloan $g^{\prime}$ & $0.0831_{-0.0036}^{+0.0061}$ & $0.1323_{-0.0058}^{+0.0192}$ & $86.30_{-0.39}^{+0.20}$ & $0.0734_{-0.0029}^{+0.0040}$ & $0.00972_{-0.00074}^{+0.00200}$ \\
\hline MPG $2.2 \mathrm{~m}$ & Sloan $r^{\prime}$ & $0.0827_{-0.0019}^{+0.00023}$ & $0.1345_{-0.0035}^{+0.00061}$ & $86.31_{-0.14}^{+0.39}$ & $0.0729_{-0.0015}^{+0.0016}$ & $0.00980_{-0.00043}^{+0.00065}$ \\
\hline MPG $2.2 \mathrm{~m}$ & Sloan $i^{\prime}$ & $0.0823_{-0.0020}^{+0.00029}$ & $0.1337_{-0.0034}^{-0.00065}$ & $85.34_{-0.17}^{+0.14}$ & $0.0726_{-0.0016}^{+0.00020}$ & $0.00970_{-0.00044}^{+0.000069}$ \\
\hline MPG $2.2 \mathrm{~m}$ & Sloan $z^{\prime}$ & $0.0865_{-0.0027}^{+0.0040}$ & $0.1424_{-0.0065}^{-0.0034}$ & $86.09_{-0.25}^{+0.17}$ & $\begin{array}{r}0.0757_{-0.0019}^{-0.00025} \\
0.016\end{array}$ & $0.01078_{-0.00075}^{+0.000144}$ \\
\hline Danish $1.54 \mathrm{~m}$ & Bessel $R$ & $0.0868_{-0.0026}^{+0.0038}$ & $0.1445_{-0.0070}^{+0.0151}$ & $86.08_{-0.25}^{+0.16}$ & $0.0758_{-0.0018}^{+0.0023}$ & $0.01095_{-0.00077}^{+0.00148}$ \\
\hline Euler $1.2 \mathrm{~m}$ & Gunn $r$ & $0.102_{-0.013}^{+0.013}$ & $0.229_{-0.080}^{+0.150}$ & $85.09_{-0.87}^{+0.86}$ & $0.0828_{-0.0047}^{+0.0021}$ & $0.0189_{-0.0074}^{+0.0127}$ \\
\hline Trappist $0.6 \mathrm{~m}$ & $I+z$ filter & $0.0854_{-0.0035}^{+0.0054}$ & $0.1310_{-0.0047}^{+0.0149}$ & $86.16_{-0.35}^{+0.20}$ & $0.0755_{-0.0028}^{+0.0038}$ & $0.00989_{-0.00065}^{+0.00164}$ \\
\hline Final results & & $0.0846 \pm 0.0012$ & $0.1379 \pm 0.0030$ & $86.20 \pm 0.07$ & $0.07455 \pm 0.00083$ & $0.01023 \pm 0.00034$ \\
\hline Hellier et al. (2012) & & & $0.1345_{-0.0019}^{+0.0048}$ & $85.8_{-0.4}^{+0.3}$ & & \\
\hline
\end{tabular}

Notes. The final parameters, given in bold, are the weighted means of the results for the datasets. Results from the discovery paper are included at the base of the table for comparison. The Euler and TRAPPIST data sets are from Hellier et al. (2012), while the others are from this work.

Table 5. Final physical properties of the WASP-67 planetary system compared with results from Hellier et al. (2012).

\begin{tabular}{llcc}
\hline \hline & & This work (final) & Hellier et al. (2012) \\
\hline Stellar mass & $M_{\mathrm{A}}\left(M_{\odot}\right)$ & $0.829 \pm 0.050 \pm 0.037$ & $0.87 \pm 0.04$ \\
Stellar radius & $R_{\mathrm{A}}\left(R_{\odot}\right)$ & $0.817 \pm 0.019 \pm 0.012$ & $0.87 \pm 0.04$ \\
Stellar surface gravity & $\log g_{\mathrm{A}}(\mathrm{cgs})$ & $4.533 \pm 0.014 \pm 0.007$ & $4.50 \pm 0.03$ \\
Stellar density & $\rho_{\mathrm{A}}\left(\rho_{\odot}\right)$ & $1.522 \pm 0.049$ & $1.32 \pm 0.15$ \\
Planetary mass & $M_{\mathrm{b}}\left(M_{\mathrm{Jup}}\right)$ & $0.406 \pm 0.033 \pm 0.012$ & $0.42 \pm 0.04$ \\
Planetary radius & $R_{\mathrm{b}}\left(R_{\mathrm{Jup}}\right)$ & $1.091 \pm 0.043 \pm 0.016$ & $1.4_{-0.2}^{+0.3}$ \\
Planetary surface gravity & $g_{\mathrm{b}}\left(\mathrm{m} \mathrm{s}^{-2}\right)$ & $8.45 \pm 0.83$ & $5.0_{-2.3}^{+1.2}$ \\
Planetary density & $\rho_{\mathrm{b}}\left(\rho_{\mathrm{Jup}}\right)$ & $0.292 \pm 0.036 \pm 0.004$ & $0.16 \pm 0.08$ \\
Planetary equilibrium temperature & $T_{\mathrm{eq}}^{\prime}(\mathrm{K})$ & $1003 \pm 20$ & $1040 \pm 30$ \\
Safronov number & $\Theta$ & $0.0457 \pm 0.0037 \pm 0.0007$ & \\
Orbital semimajor axis & $a(\mathrm{au})$ & $0.0510 \pm 0.0010 \pm 0.0008$ & $0.0517 \pm 0.0008$ \\
Age & $\mathrm{Gyr}$ & $8.7_{-7.3-12.7}^{+15.5}$ & $2.0_{-1.0}^{+1.6}$ \\
\hline
\end{tabular}

Notes. Two sets of errorbars are given for the results from the current work, the former being statistical and the latter systematic.

set of theoretical stellar models for the calculated stellar mass and $\left[\frac{\mathrm{Fe}}{\mathrm{H}}\right]$. Statistical errors were propagated by a perturbation analysis, and the overall best fit was found by evaluating results for a grid of ages. We assessed the contribution of systematic errors from theoretical stellar models by running solutions for five different grids of models (Claret 2004a; Demarque et al. 2004; Pietrinferni et al. 2004; VandenBerg et al. 2006; Dotter et al. 2008). The final set of physical properties was calculated by taking the unweighted mean of the five sets of values found from the different stellar models, and the systematic errors were taken to be the maximum deviation of a single value from the mean. The physical parameters of the WASP-67 planetary system are given in Table 5.

Table 5 also shows the values obtained by Hellier et al. (2012) for comparison. We find a smaller radius for the star, which is attributable to the better constraint on the stellar density from our high-precision light curves. We also obtain a significantly smaller planetary radius and, hence, a larger surface gravity and density. This is due partly to the smaller stellar radius combined with a comparable measurement of $k$ (Table 4) and partly to an inconsistency among the $R_{\mathrm{A}}, R_{\mathrm{b}}$, and $k$ values found by Hellier et al. (2012). The latter issue arises because Hellier et al. (2012) quote the median value of each fitted parameter from Markov Chain Monte Carlo simulations rather than giving the set of parameters corresponding to the single best-fitting link in the Markov chain (D. R. Anderson, priv. comm.).

\section{Variation of the planetary radius with wavelength}

If it were not for the difficulty of measuring its radius, WASP$67 \mathrm{~b}$ would be a good target for studies of the planetary atmosphere due to its low surface gravity. However, its moderate equilibrium temperature $\left(T_{\text {eq }}^{\prime}=1003 \pm 20 \mathrm{~K}\right)$ indicates that the planet should belong to the pL class (Fortney et al. 2008), implying that we do not expect to measure large variations of the planet radius with wavelength. As the GROND instrument is able to cover different optical passbands, we used our data to probe the terminator region of the planetary atmosphere.

Following our method in previous works (Southworth et al. 2012; Mancini et al. 2013b), we re-fitted the GROND light curves with all parameters, except $k$, fixed to the final values given in Table 4. This approach maximizes the precision of estimations of the planet/star radius ratio by removing common sources of uncertainty. We find the following values: $k=$ $0.1369 \pm 0.0063$ for $g^{\prime}, k=0.1384 \pm 0.0026$ for $r^{\prime}, k=$ $0.1381 \pm 0.0024$ for $i^{\prime}$, and $k=0.1387 \pm 0.0023$ for $z^{\prime}$. These results are shown in Fig. 5, where the vertical errorbars represent the relative errors in the measurements and the horizontal errorbars show the FWHM transmission of the passbands used. For illustration, we also show the predictions from a model atmosphere calculated by Fortney et al. (2010) for a Jupitermass planet with a surface gravity of $g_{\mathrm{b}}=10 \mathrm{~m} \mathrm{~s}^{-2}$, a base radius of $1.25 R_{\mathrm{Jup}}$ at $10 \mathrm{bar}$, and $T_{\mathrm{eq}}^{\prime}=1000 \mathrm{~K}$. The opacity of 


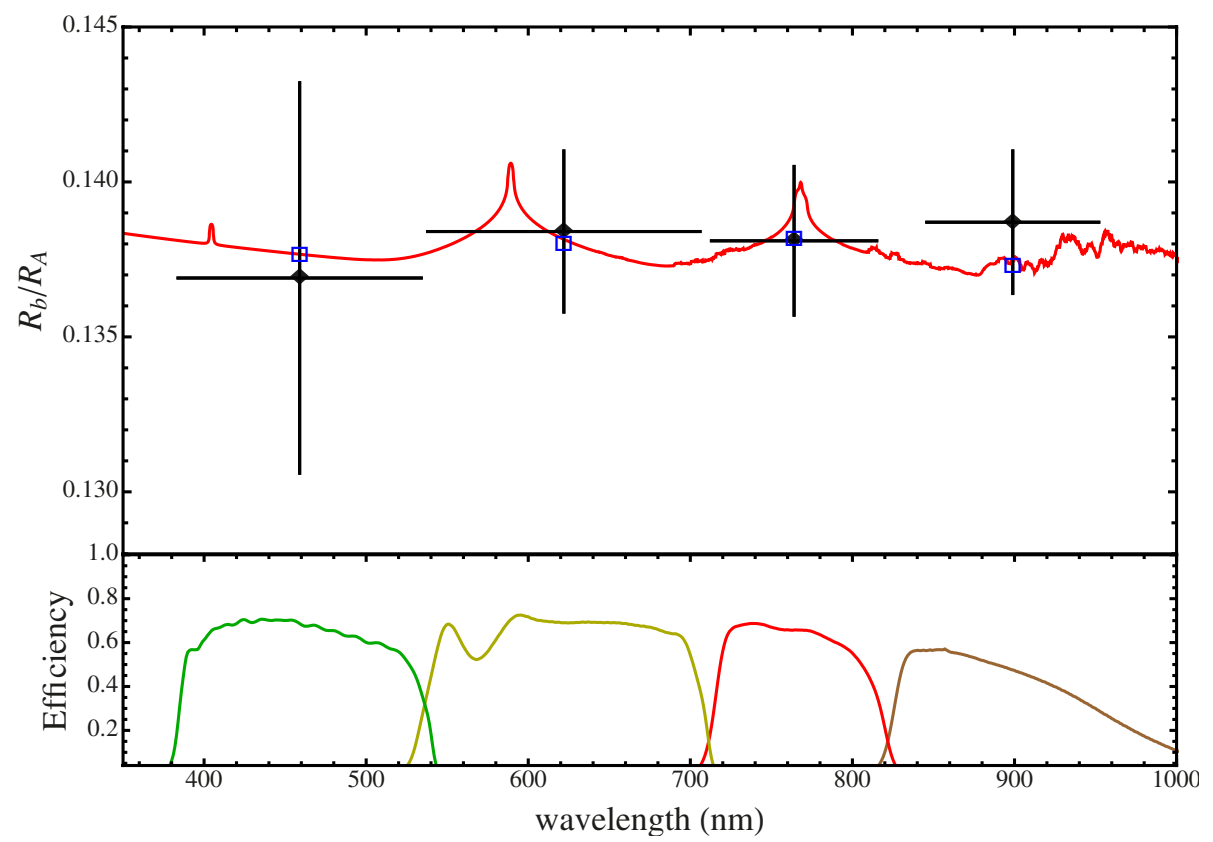

Fig. 5. Variation of the planetary radius, in terms of planet/star radius ratio, with wavelength. The black diamonds are from the transit observations performed with GROND. The vertical bars represent the errors in the measurements and the horizontal bars show the FWHM transmission of the passbands used. The observational points are compared with a synthetic spectrum (see text for details). Total efficiencies of the GROND filters are shown in the bottom panel. The blue boxes indicate the predicted values for the model integrated over the passbands of the observations.

strong-absorber molecules, such as gaseous titanium oxide ( $\mathrm{TiO})$ and vanadium oxide (VO), was removed from the model. Our experimental points agree with the prominent absorption features of the model (sodium at $\sim 590 \mathrm{~nm}$ and potassium at $\sim 770 \mathrm{~nm}$ ) and, being compatible with a flat transmission spectrum, do not indicate any large variation of the WASP-67 b's radius.

\section{High-resolution image}

Eclipsing binary star systems are a common source of false positives for transiting planets detected by wide-field photometry. The host star can have a gravitationally bound companion, or its light can be contaminated by a background eclipsing binary, which is coincidentally at the same sky position. Both cases can mimic a planetary-transit signal. Faint, close stars may also contaminate the PSF of the target star, thus slightly lowering the depth of the transit and causing us to underestimate the radius of both the TEP and its host star. Finally, these faint nearby stars could also affect the radial velocity measurements of the star and, thus, the measured mass of the planet (e.g. Buchhave et al. 2011).

To check if WASP-67 A is contaminated by any faint companion or background stars, we observed it on 2014/04/21 with the Andor Technology iXon+ model 897 EMCCD Lucky Camera mounted at the Danish $1.54 \mathrm{~m}$ telescope. The imaging area of this camera is $512 \times 512$ pixels, and each $16 \mu \mathrm{m}$ pixel projects to 0 . $^{\prime} 09$ on the sky, which gives a $45 \times 45 \operatorname{arcsec}^{2}$ field of view. The camera has a special long-pass filter with a cut-on wavelength of $650 \mathrm{~nm}$, which corresponds roughly to a combination of the SDSS $i^{\prime}+z^{\prime}$ filters (Skottfelt et al. 2013).

Figure 6 shows the resulting image. WASP-67 A is the bright star in the centre of the image. Figure 7 shows the central region of the image, and it can be seen that two stars (A and B) occur approximately $4.5^{\prime \prime}$ and $6.0^{\prime \prime}$ northeast of WASP-67 A. The plate scales and inner apertures of DFOSC and GROND (Table 1) are such that both stars are inside the defocused PSFs of WASP-67. However, they are much fainter than WASP-67 A with $\Delta\left(i^{\prime}+z^{\prime}\right)=7.6 \mathrm{mag}$ and $7.9 \mathrm{mag}$, respectively. They, therefore, contribute only $0.1 \%$ and $0.07 \%$ of the total flux in each image, so have a negligible effect on our results.

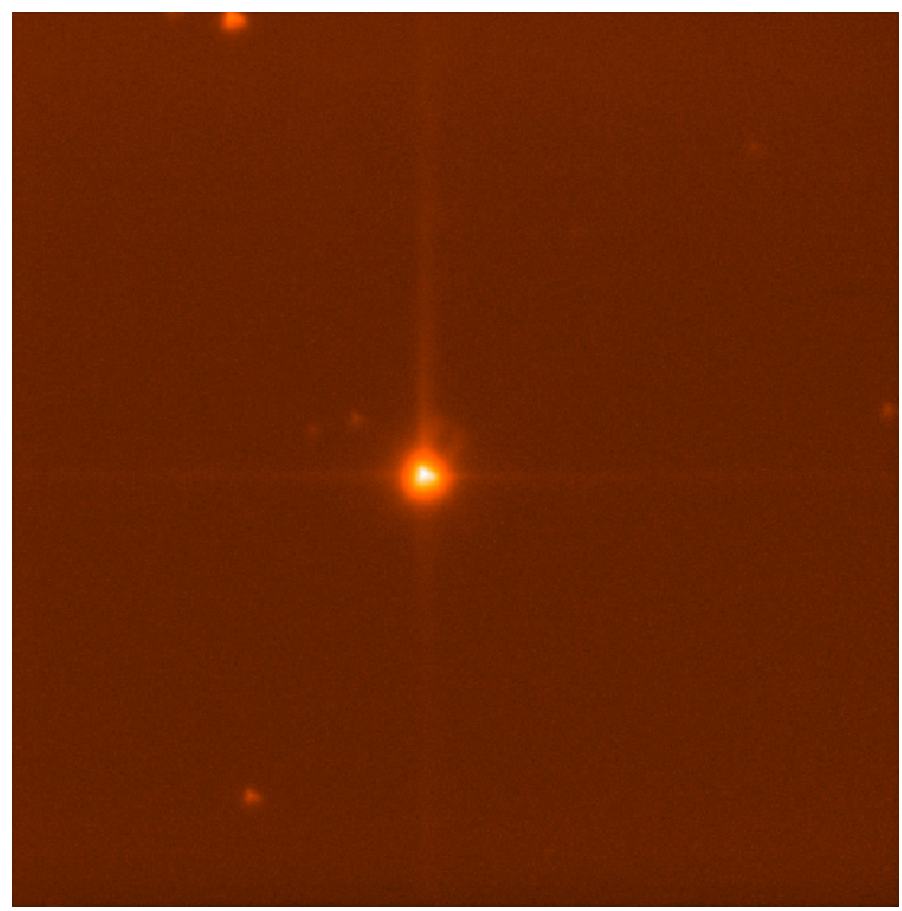

Fig. 6. Lucky Camera image of WASP-67. The image size is $45 \times$ $45 \operatorname{arcsec}^{2}$ and is shown in a logarithmic flux scale with north up and east to the left. The FWHM of the image is 0.54 . The triangular PSF comes from the telescope in very good seeing. The extra flux north-west of WASP-67 A is not a real contaminating flux source but an optical ghost from the star caused by internal reflections within the beamsplitters.

In the eventuality that the two faint nearby stars are intrinsically very blue objects, they could have affected our $g^{\prime}$-band observations by more than the amount given above. Measurement of a colour index from multiple high-resolution images would allow this possibility to be investigated. As a worst-case scenario, if both contaminants have $T_{\text {eff }}=30000 \mathrm{~K}$ and are located at such as distance as to contribute $0.1 \%$ of the flux in the Lucky Camera 


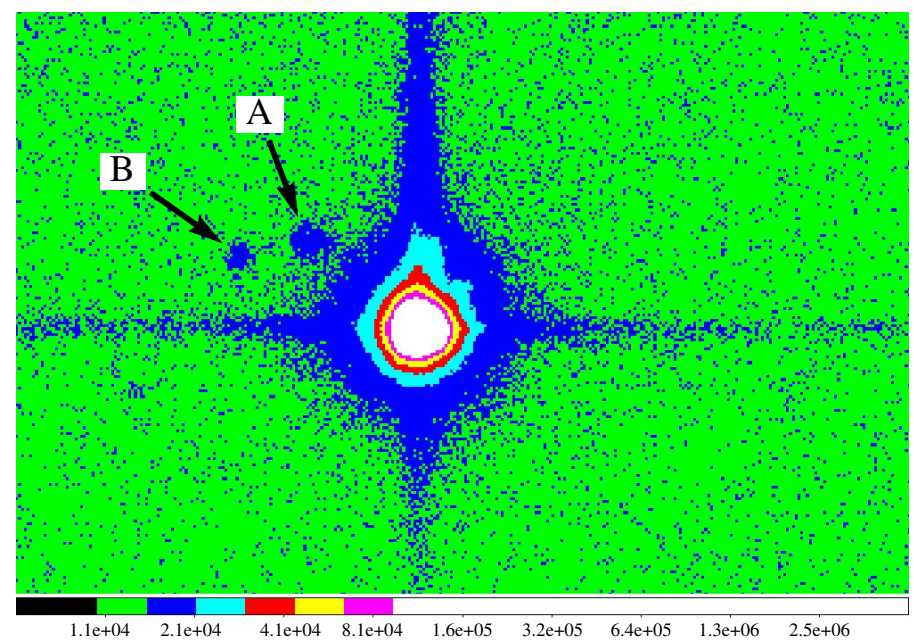

Fig. 7. Central part of the Lucky Camera image in Fig. 6. The image is shown in with a logarithmic flux scale with north up and east to the left. Two faint stars, A at $\sim 4.4^{\prime \prime}$ and B at $\sim 6.0^{\prime \prime}$ north-east of WASP$67 \mathrm{~A}$, are evident. Values in the colour bar refer to the number of counts in ADU.

passband, the contamination in the $g^{\prime}$-band would be $1.1 \%$. This figure remains too small to be important to the current analysis.

\section{Kepler-K2 observations}

A more extensive study of the WASP-67 planetary system is anticipated, as this object will be observed by the Kepler satellite during its K2 phase. To explore the impact of these forthcoming observations, we have generated a synthetic light curve matching the K2 data characteristics and subjected it to the same modelling process as for the real data presented in the current work.

We calculated a model light curve for the best-fitting photometric parameters (Table 4) using JKTEBOP and for quadratic LD coefficients appropriate for the $K_{p}$ passband (Claret 2004b). This was extended over the full duration of the observations for field \#2 (as the schedule for field \#7 is not yet set) and numerically integrated to the duration of the short-cadence $(58.8 \mathrm{~s}$ ) and long-cadence $(29.4 \mathrm{~min})$ data types obtained by Kepler. Gaussian random noise was added to each data point equivalent to a scatter of 100 parts per million per six-hour time interval (Howell et al. 2014, their Fig. 10). Data points outside orbital phases -0.02 to 0.02 were discarded for computational convenience.

The synthetic light curves were fitted with JKTEBOP using the same treatment as our real data sets for WASP-67, with the exception that we numerically integrated the model for the longcadence simulated data to match its sampling rate (Southworth 2011). We find that the uncertainties in the resulting photometric parameters are quite similar between the two cadences, which is due to the relatively smooth brightness variation through the partial eclipse of WASP-67. They are also similar to those of our final parameters in Table 4, suggesting that the Kepler data will not allow a substantial improvement in the measured physical properties of WASP-67. This result was unexpected but can be explained by the larger scatter of the Kepler data $(0.83 \mathrm{mmag}$ for short-cadence) versus our best light curves (see Table 1).

One possibility, which is much better suited to K2 observations, is the detection of the rotational period of WASP-67 A due to spot-induced brightness modulations. WASP-67 A is a cool star $(5200 \mathrm{~K})$, but no spot modulation was detected in the SuperWASP light curve to a level of roughly $1 \mathrm{mmag}$. The data acquired by $\mathrm{K} 2$ may allow the rotational period to be estimated, which is useful for dynamical and tidal studies.

\section{Summary and conclusions}

We have presented the first follow-up study of the planetary system WASP-67, which is based on the analysis of five new light curves of two transit events of WASP-67 b. The first transit was observed simultaneously with GROND through Sloan $g^{\prime}, r^{\prime}, i^{\prime}$, $z^{\prime}$ filters; the second was observed in Bessell- $R$ with DFOSC. The transits were monitored roughly one and two years, respectively, after the reference epoch used by Hellier et al. (2012). Both transit events were observed in telescope-defocusing mode, resulting in a photometric precision of $0.48-1.08$ mmag per observation. We modelled our new and two published datasets using the JKTEBOP code. By estimating the impact parameter $b$ and the ratio of the planet/star radii, we found that the criterion for a grazing eclipse, $b+k>1$, is satisfied for all the light curves, confirming that the eclipse is grazing.

We used the results of the light-curve analysis to substantially improve the measurements of the physical properties of the planet and its host star (Table 5). Compared to the discovery paper (Hellier et al. 2012), we find a significantly smaller radius and a greater density for WASP-67 b. We obtain $R_{\mathrm{b}}=1.091 \pm$ $0.046 R_{\text {Jup }}$ versus $1.4_{-0.2}^{+0.3} R_{\text {Jup }}$, and $\rho_{\mathrm{b}}=0.292 \pm 0.036 \rho_{\text {Jup }}$ versus $0.16 \pm 0.08 \rho_{\text {Jup }}$. Our revised physical properties move WASP$67 \mathrm{~b}$ into a quite different region of parameter space. Figure 8 shows the change in position in the planet mass-radius plot (top panel) and in the planet mass-density plot (bottom panel). The revised positions are marked with a green circle, while the red circle indicates the old values from Hellier et al. (2012). The values of the other TEPs were taken from the TEPCat catalogue ${ }^{7}$. For illustration, the bottom panel of Fig. 8 also shows $10 \mathrm{Gyr}$ isochrones of exoplanets at $0.045 \mathrm{AU}$ orbital separation from a solar analogue (Fortney et al. 2007). The plot suggests that WASP-67 b should have a more massive core than previously thought.

As an additional possibility offered by the GROND data, we made an attempt to investigate possible variations of the radius of WASP-67 b in different optical passbands. Our experimental points are compatible with a flat transmission spectrum and do not indicate any large variation of the planet's radius. The gradual increase of the transit depth moving from the GROND $g^{\prime}$ to $z^{\prime}$ band, which is opposite to the case for higher-inclination systems, is explicable in that WASP-67 b only produces grazing eclipses. Due to stronger limb darkening, these are shallower in the blue bands than in the red ones.

Acknowledgements. This paper is based on observations collected with the MPG $2.2 \mathrm{~m}$ and the Danish $1.54 \mathrm{~m}$ telescopes, both located at ESO Observatory in La Silla, Chile. Operation of the Danish telescope is based on a grant to U.G.J. by the Danish Natural Science Research Council (FNU). GROND was built by the high-energy group of MPE in collaboration with the LSW Tautenburg and ESO, and is operated as a PI-instrument at the MPG $2.2 \mathrm{~m}$ telescope. We thank David Anderson and Coel Hellier for useful discussions, and the referee for a helpful report. J.S. (Keele) acknowledges financial support from STFC in the form of an Advanced Fellowship. C.S. received funding from the European Union Seventh Framework Programme (FP7/2007-2013) under grant agreement No. 268421. M.R. acknowledges support from FONDECYT postdoctoral fellowship $\mathrm{N}^{\circ} 3120097$. T.C.H. would like to acknowledge KASI grant \#2014-1-400-06. H.K. acknowledges support by the European Commission under the Marie Curie Intra-European Fellowship Programme in FP7. S.-H.G. and X.-B.W. would like to thank the financial support from National Natural Science Foundation of China (No. 10873031) and Chinese Academy of Sciences (project KJCX2-YW-T24). O.W. thanks the Belgian National Fund for Scientific

http://www .astro.keele.ac.uk/jkt/tepcat/ 

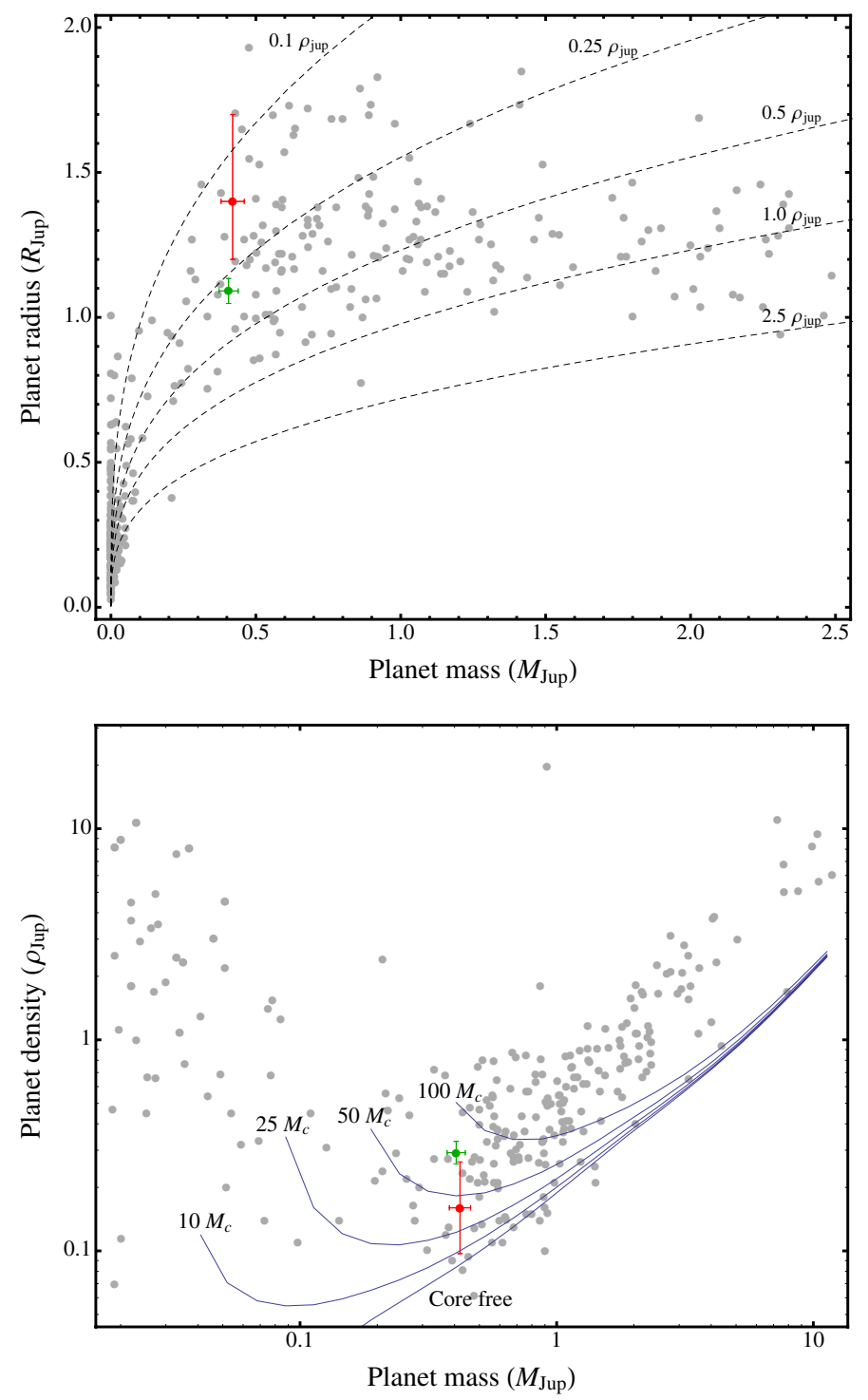

Fig. 8. Top panel: masses and radii of the known TEPs. The grey points denote values taken from TEPCat. Their error bars have been suppressed for clarity. WASP-67 b is shown with red (Hellier et al. 2012) and green (this work) points with error bars. Dotted lines show where density is $2.5,1.0,0.5,0.25$ and $0.1 \rho_{\text {Jup }}$. Bottom panel: the massdensity diagram of the currently known transiting exoplanets (taken from TEPCat). Four planetary models with various core masses and another without a core (Fortney et al. 2007) are plotted for comparison.
Research (FNRS). J.S. and O.W. acknowledge support from the Communauté française de Belgique - Actions de recherche concertées - Académie universitaire Wallonie-Europe. K.A., M.D. and M.H. acknowledge grant NPRP-09-4761-78 from the Qatar National Research Fund (a member of Qatar Foundation). This publication was aided by NPRP grant \# X-019-1-006 from the Qatar National Research Fund (a member of Qatar Foundation). The reduced light curves presented in this work will be made available at the CDS (http:// cdsweb.u-strasbg.fr/). The following internet-based resources were used in research for this paper: the ESO Digitized Sky Survey; the NASA Astrophysics Data System; the SIMBAD data base operated at CDS, Strasbourg, France; and the arXiv scientific paper preprint service operated by Cornell University.

\section{References}

Anderson, D. R., Barros, S. C. C., Boisse, I., et al. 2011, PASP, 123, 555 Béky, B., Bakos, G. Á., Hartman, J., et al. 2011, ApJ, 734, 109

Buchhave, L. A., Latham, D. W., Carter, J. A., et al. 2011, ApJS, 197, 3 Carter, J. A., \& Winn, J. N. 2009, ApJ, 704, 51

Claret, A. 2004a, A\&A, 424, 919

Claret, A. 2004b, A\&A, 428, 1001

Demarque, P., Woo, J.-H., Kim, Y.-C., Yi, S. K. 2004, ApJS, 155, 667

Dotter, A., Chaboyer, B., Jevremović, D., et al. 2008, ApJS, 178, 89

Dominik, M., Jørgensen, U. G. , Rattenbury, N. J., et al. 2010, Astron. Nachr., 331,671

Fortney, J. J., Marley, M. S., \& Barnes J. W. 2007, ApJ, 659, 1661

Fortney, J. J., Lodders, K., Marley, M. S., \& Freedman R. S. 2008, ApJ, 678 , 1419

Fortney, J. J., Shabram, M., Showman, A. P., et al. 2010, ApJ, 709, 1396

Gillon, M., Pont, F., Moutou, C., et al. 2006, A\&A, 459, 249

Gibson, N. P., Pollacco D., Simpson E. K., et al. 2008, A\&A, 492, 603

Greiner, J., Bornemann, W., Clemens, C., et al. 2008, PASP, 120, 405

Hellier, C., Anderson, D. R., Collier Cameron, A., et al. 2012, MNRAS, 426, 739

Howell, S. B., Sobeck, C., Haas, M., et al. 2014, PASP, 126, 398

Knutson, H. A., Charbonneau, D., \& Noyes, R. W. 2007, ApJ, 655, 564

Mancini, L., Southworth, J., Ciceri, S., et al. 2013a, A\&A, 551, A11

Mancini, L., Nikolov, N., Southworth, J., et al. 2013b, MNRAS, 430, 2932

Mancini, L., Ciceri, S., Chen, G., et al. 2013c, MNRAS, 436, 2

Mancini, L., Southworth, J., Ciceri, S., et al. 2014, A\&A, 562, A126

Nikolov, N., Henning, Th., Koppenhoefer, J., et al. 2012, A\&A, 539, 159

Müller, H. M., Huber, K. F., Czesla, S., et al. 2013, A\&A, 560, A112

Pietrinferni, A., Cassisi, S., Salaris, M., \& Castelli, F. 2004, ApJ, 612, 168

Pollacco, D. L., Skillen, I., Collier Cameron, A., et al. 2006, PASP, 118, 1407

Skottfelt, J., Bramich, D. M., Figuera Jaimes, R., et al. 2013, A\&A, 553, A111

Smalley, B., Anderson, D. R., Collier Cameron, A., et al. 2011, A\&A, 526, A130

Southworth, J. 2008, MNRAS, 386, 1644

Southworth, J. 2011, MNRAS, 417, 2166

Southworth, J. 2012, MNRAS, 426, 1291

Southworth, J., Hinse, T. C., Jørgensen, U. G., et al. 2009, MNRAS, 396, 1023 Southworth, J., Mancini, L., Maxted, P. F. L., et al. 2012, MNRAS, 422, 3099

VandenBerg, D. A., Bergbusch, P. A., \& Dowler, P. D. 2006, ApJS, 162, 375

Winn J. N. 2010, in Exoplanet, ed. S. Seager (The University of Arizona Press), 56

Winn, J. N., Holman, M. J., Torres, G., et al. 2008, ApJ, 683, 1076 